\title{
A relaxationless demonstration of the Quantum Zeno Paradox on an individual atom
}

\author{
Chr. Balzer, Th. Hannemann, D. Reiß, Chr. Wunderlich, W. Neuhauser, P.E.Toschek \\ Institut für Laser-Physik, Luruper Chaussee 149, D-22761 Hamburg, Germany
}

25.03.2002

\begin{abstract}
The driven evolution of the spin of an individual atomic ion on the ground-state hyperfine resonance is impeded by the observation of the ion in one of the pertaining eigenstates. Detection of resonantly scattered light identifies the ion in its upper "bright" state. The lower "dark" ion state is free of relaxation and correlated with the detector by a null signal. Null events represent the straightforward demonstration of the quantum Zeno paradox. Also, high probability of survival was demonstrated when the ion, driven by a fractionated $\pi$ pulse, was probed and monitored during the intermissions of the drive, such that the ion's evolution is completely documented.
\end{abstract}




\section{Introduction}

Among the most fundamental questions posed in the continued debate on the foundations of quantum mechanics are three issues: (1) The nature of a quantum system in the state of temporal evolution versus being in one of its eigenstates. (2) The nature of quantum-mechanical measurement. (3) The emergence of the classical world out of many interacting quantum systems. Related to all these issues is a long-standing particular problem, namely the temporal evolution of a quantum system under continuous or repeated observation. From the characteristics of the unitary rotation in phase space and von Neumann's state reduction it had been concluded that the reiterated detection of a particular observable of the system being involved in a quantum system's evolution keeps projecting the system back into the state in which it was initially prepared, and in the limit takes the evolution to a halt [1-3]. More recently it has been argued that in order not to confuse this effect of measurement with the effect of dynamical intervention in the observed system, by environment or meter, this quantum system under scrutiny must consist of an individual entity: On an ensemble, those two different kinds of effects cannot be distinguished in principle [4-6]. Moreover, the demonstration of the strangest and most perplexing features of the measurement process was said to require non-local interaction indicated by a null result ("quantum Zeno paradox", QZP [7]).

Recently, an experiment on the quantum evolution of an individual atomic ion has been reported that satisfies these preconditions [8]. The inhibition of the ion's evolutionon a weak resonance upon repeated probing its state by reiterated attempts of making the ion scatter resonance light was demonstrated. This evidence was derived from the statistics of uninterrupted sequences of equal results, i.e., either all results in a sequence signal "scattered light on", or all "off". The frequencies of occurrence of such recorded sequences were determined. They agreed with the joint probabilities for survival of the ion in its state upon $1,2,3, \ldots$ attempts of excitation or deexcitation, calculated under the condition that the ion, after each light-driven period of evolution, is set back, by the probing, to its initial eigenstate. In particular, the "off" results that were correlated with the ion excited into a metastable electronic state represent a remarkable demonstration, since the corresponding measurements satisfy the condition of quantum non-demolition $[4,8,9]$. On the other hand, although the decay of that "dark" state via an $E 2$ line is rather weak, the data nevertheless require the consideration of relaxation with their quantitative evaluation that is found some-

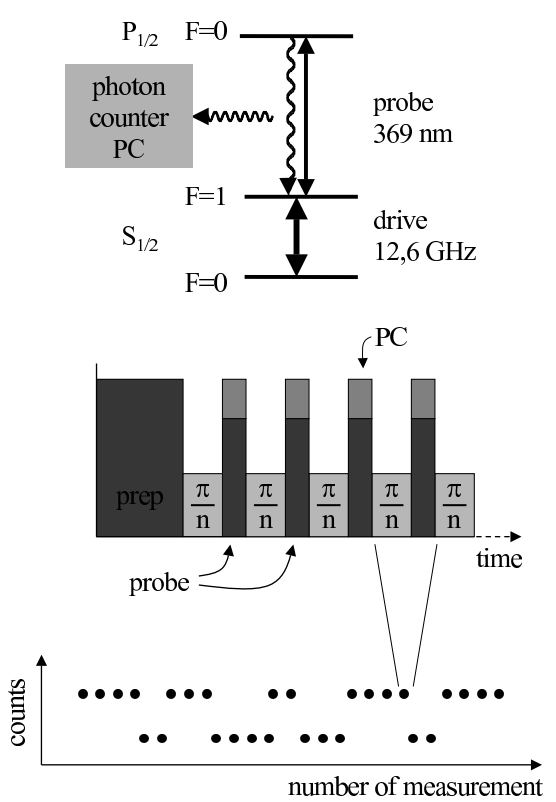

Figure 1: Relevant energy levels of ${ }^{171} \mathrm{Yb}^{+}$ion, microwave driving radiation, resonant probe light, and detection of resonance fluorescence (top). Temporal schedule of preparation and measurements (centre). Trajectory of results (schematic) made up of sequences of "on" and "off" results alternating.

what involved.

We report on an alternative, straightforward microwave-optical double-resonance experiment on an individual quantum system that avoids any complication. Pulses of $12.6-\mathrm{GHz}$ microwave radiation drive the ground-state hyperfine transition of a single trapped ${ }^{171} \mathrm{Yb}^{+}$ion. Excitation of this ion to its $F=1$ state - if having happened - is monitored by laser pulses alternating with the driving pulses: some of their light is scattered off the ion, on the $S_{1 / 2}(F=1)-P_{1 / 2}$ resonance line. In this quantum system, the "dark" state is the lower $F=0$ level of the ion's electronic ground state and as such free of intrinsic relaxation. Phase fluctuation of the microwave is negligible. The "bright" state is the upper $F=1, m_{F}=0$ ground-state level, which is also free of relaxation, but optical pumping into the Zeeman sublevels $m_{F}= \pm 1$ by the probe light may decouple the ion from the microwave driving and mimic energy relaxation [10]. The relevant levels of the ion, driving and probing light, and the laser-excited and detected resonance fluorescence are schematically outlined in Fig 1 (top).

From recorded trajectories of the results of reiterated measurements, the distributions of sequences of identical results were determined. They fit in with the distributions of the calculated joint probabilities of survival when assuming state reduction to happen with the probing. - An alternative strategy involved $n$-fold irradiation of the ion by driving 
$\pi / n$-pulses, intertwined by probe pulses. Recording of the corresponding bursts of scattered probe light - or their absence - allows complete characterization of the ion's evolution along the trajectory of measurements. This strategy amounts to a complete, or "selective" measurement of the ion's probability of survival in its eigenstate. This probability increases with $n$, and in particular the "off" sequences thus demonstrate the QZP.

\section{Experimental}

The experimental concept is close to a previously used one [11]. However, the source of the probe light has been modified: Frequency-doubled light of a Ti:sapphire laser at $369 \mathrm{~nm}$ wavelength with about $100 \mathrm{kHz}$ bandwidth, some $10 \mathrm{MHz}$ down-tuned from resonance, was scattered on the $S_{1 / 2}-P_{1 / 2}$ ionic resonance line. Microwave and laser parameters as well as data acquisition were controlled in real time. The ion was occasionally pumped into the metastable $D_{3 / 2}$ level, but it was immediately repumped, into the ground state, by the 935-nm light of a diode laser. Residual micro-motion of the ion was monitored by phase-sensitive detection of motional fluorescence modulation, and eliminated. Thus, the ion was localized in the node of the trapping field with less than $10 \mathrm{~nm}$ deviation. Its steady-state vibrational temperature was at the Doppler cooling limit, below $1 \mathrm{mK}$, and well inside the Lamb-Dicke regime.

With the laser light off, the ion was irradiated by microwave pulses of duration $\tau$ and Rabi frequency $\Omega$, such that the pulse area was $\theta=\Omega \tau=$ $\pi / n$, where $n$ is a small integer. Particular care was taken with the precise setting of the resonance frequency and of $\theta$. Any unwanted detuning of the microwave frequency from exact resonance was uncovered when the ion was driven by double pulses, and afterwards probed by a resonant laser pulse, according to the temporal version of Ramsey's technique [11]. The results of some 500 of these measurements with stepwise incremented temporal separation make a trajectory. With 50 samples of such trajectories superimposed, interference fringes herald residual precession of the ion's spin and the concomitant detuning of the microwave from resonance, that was to be eliminated.

The pulse area was set by stepwise incrementing the length of single driving pulses, being followed by a pulse of probe light (Fig. 2). Superimposed trajectories of results reveal nutational oscillation of the ion's spin whose phase is identified with the pulse area $\theta$, such that the desired pulse length can be precisely preset, to a well-defined fraction of $\pi$, in contrast with the previous experiment [8].

In the actual measurements, microwave driving

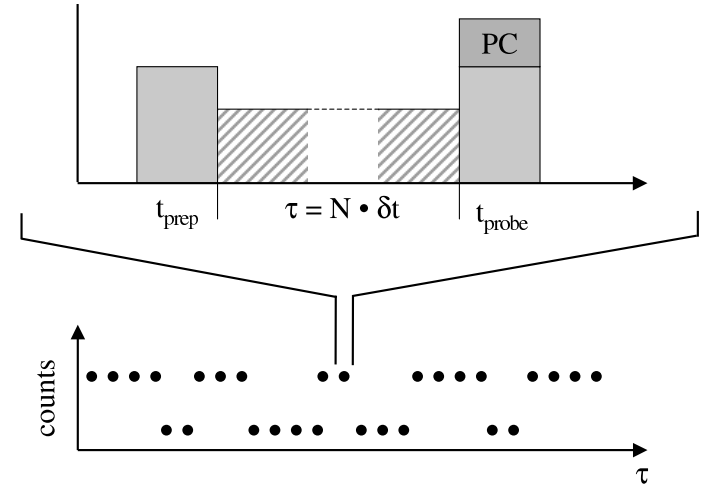

$50 \times$

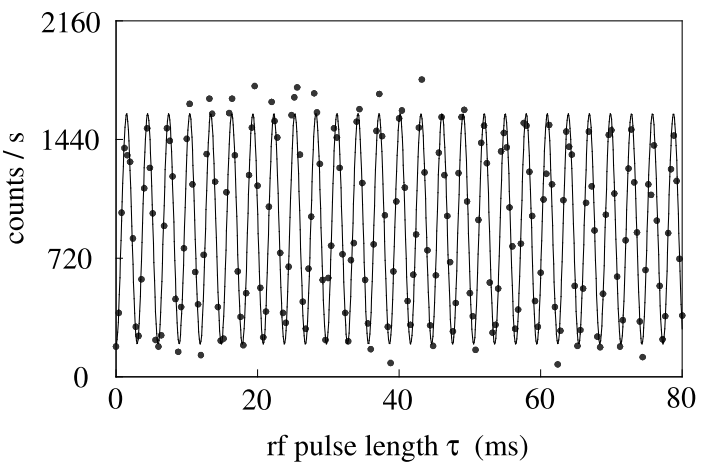

Figure 2: Setting the pulse area of the driving radiation: A test measurement on the single ${ }^{171} \mathrm{Yb}^{+}$ion is made up of a laser pulse that pumps the ion into the $F=0$ level of the ground state, a microwave driving pulse whose duration is stepwise increased in the next measurement, and a probe pulse with simultaneous detection of resonant scattering. A series of 500 measurements make a trajectory; 50 superimposed trajectories show Rabi pulsations from the ion's optical nutation. The phase of pulsation is identified with the area $\theta=\Omega \tau$ of the corresponding driving pulse. In a similar way, the resonant tuning of the drive was tested in a Ramseytype scheme, when the driving pulse was replaced by two 2-ms-long pulses separated by stepwise increased intermittance.

pulses of duration $\tau$ were separated by 1.5 -ms long probe pulses of resonant light applied to the ion that were generated when gating the cw laser light by an acousto-optical deflector. The pulse area was $2 \pi$ when $\tau=4.9 \mathrm{~ms}$. The scattered light was recorded by a photon counter gated open in synchronism with the probe pulses (Fig. 1, centre). When a laser pulse excited a burst of resonance scattering, the ion was considered to be on the $F=1$ hyperfine level of its ground state; when no resonance fluorescence was excited, the ion was supposed to be on level $F=0$. Each pair of driving and probing pulses represents the coherent preparation of - in general - a superposition ground state, and the subsequent read out of one of the pertaining eigenstates. Series 
of 10000 of such measurements resulted in the generation of corresponding trajectories of results made up of alternating "waiting intervals" each of which contains a quasi-random number of equal "on" or "off" results (Fig. 1, bottom). The statistical distributions of all sequences of equal results contained in these waiting intervals was evaluated for an analysis of the retarding effect of the measurements upon the quantum evolution of the ion on the ground-state hyperfine resonance.

\section{$3 \quad$ Statistics of sequences of equal results}

From the data of the observed trajectories, one wants to derive a measure for the probability of the ion's survival, during $q$ sequential attempts of driving and probing, in its eigenstate "on" (1) or "off" (0). This probability is identified with the normalized frequency of occurrence, $U(q)$, of that sequence in a trajectory, a good approximation with a long trajectory. Matching a value $V$ calculated under the condition of the ion's evolution being frustrated by measurements,

$$
U(q) / U(1)=V(q-1),
$$

verifies QZE. The probability $V$ is easily calculated if we assume that the driven evolution of the ion is interrupted by the probing that leaves the ion in an eigenstate. In any sequence of equal results, this is the same state - namely $|1\rangle$, correlated with "on", or $|0\rangle$, correlated with "off" - where the ion had been observed by the first measurement of the sequence [8]. Since the probability of survival after the action of one resonant driving pulse of area $\Omega \tau$ is $p=\cos ^{2}(\Omega \tau / 2)$ [12], the conditional probability of $q$-times survival, under the above assumption, is simply $V(q)=p^{q}$. Note that we need not consider, with the data evaluation, the complications brought about by relaxation processes, since there is no intrinsic relaxation involved with the ground-state hyperfine transition. Thus, the probability $p$ of the ion staying in its same state as observed before, under the action of a driving pulse, is not supposed to differ in the two states, $p_{0}=p_{1}$. However, although state 0 is indeed free of relaxation, the effect of optical pumping among the Zeeman levels of state 1 by the probe light provides the ion with some effective energy relaxation [10].

Fig. 3 shows statistical distributions of the "off" sequences, namely $U(q) / U(1)$, on a logarithmic scale, for nominally $n=1,2,5$, i.e., $\theta=\pi, \pi / 2, \pi / 5$, and $\theta=2 \pi-0.1$. Also shown are lines representing $V(q-1)$ that are made to fit the data by vary$\operatorname{ing} \theta$. The fitting procedure is very sensitive: deviations $\delta \theta=10^{-5}$ are recognizable. It turns out,

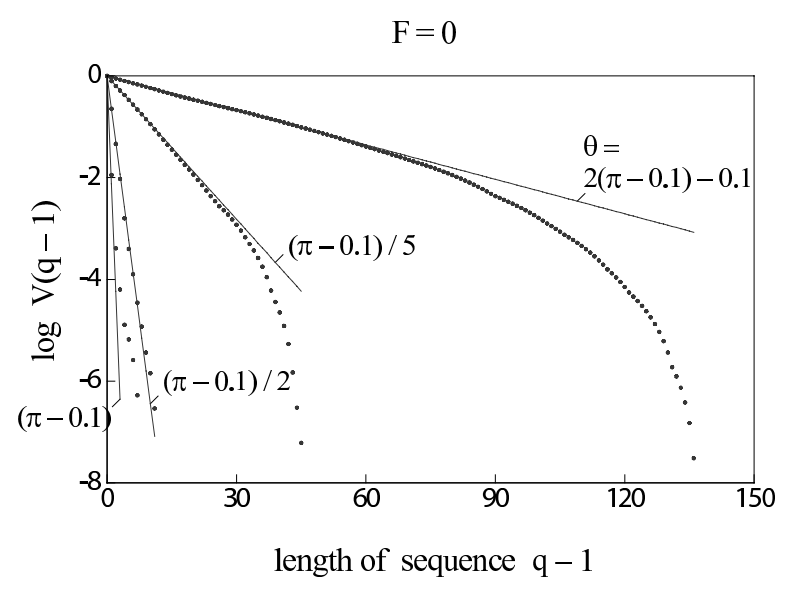

Figure 3: Probability $U(q) / U(1)$ of uninterrupted sequences of $q$ results all of them "off", when ion was initially prepared in the "off" state $(F=0)$. The lines show the distribution of probability $V(q-1)$ for the ion not undergoing a flip of its nuclear spin to the "on" state during the entire sequence. Length of trajectories: 2000 measurements of $4.9 \mathrm{~ms}$ driving time and $2 \mathrm{~ms}$ probing time. See text.

that the preset areas of the microwave $\pi$ pulses deviated from their nominal values by $3 \%$. At large $q$, the data show deficiency of long sequences, marking slightly excessive excitation to the $F=1$ level. This feature indicates some dephasing of the driven spin dynamics to happen on the time scale of seconds.

The distributions of the sequences of "on" results (not shown) also follow straight lines on the logarithmic scale and indicate that the probability $p_{1}=p_{0}$ does not depend on $q$. However, the variation of $U(q) / U(1)$ with $\theta$ is relatively small. These features show, even on the time scale of only a few measurements, the effective relaxation by Zeeman pumping and the corresponding decoupling of the ion from the driving microwave radiation. Thus, excessive probability of long "on" sequences may result.

\section{Interaction with fractionated $\pi$ pulses}

We have also implemented an alternative strategy of measurement. After a preparatory laser pulse that pumps the ion into the $F=0$ ground state, the ion was irradiated by a series of $n$ driving pulses of area $\pi / n$. This fractionated excitation would result in a complete $\pi$ flop of the ion into state $F=1$, provided that dephasing is safely negligible (Fig. 4(a) and (b)). Pulses of probe light illuminated the ion during the $n-1$ intermissions between the individual driving pulses, and the photon counter was synchronously gated open in order to register light 
a)

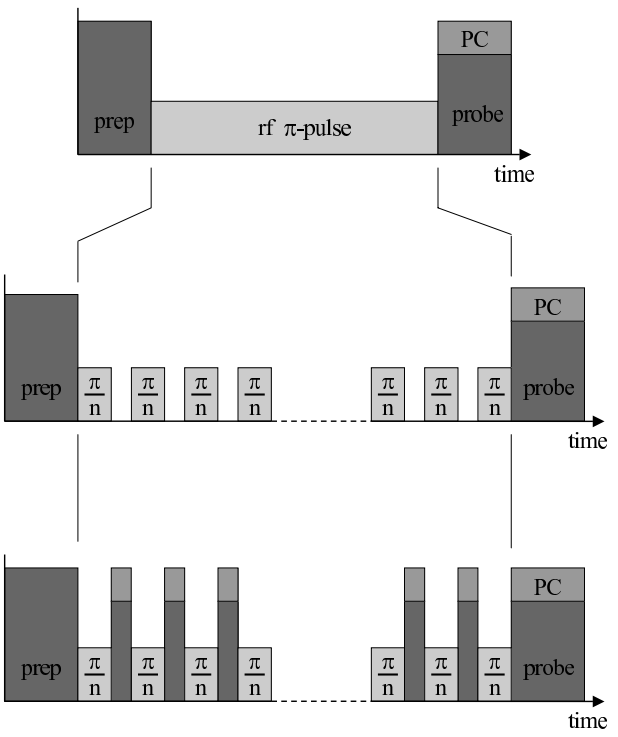

Figure 4: Scheme of measurement with one driving $\pi$ pulse (a). Same with fractionated $\pi$ pulse $(n$-times $\pi / n)$, no intermediate probing (b). Same, but $\pi / n$ pulses alternating with probe pulses (c). PC: photon counting.

scattering as the signature of ion excitation after any one of the driving pulses. The result - scattered light on or off - generated by the final ( $n$ th) probe pulse was separately registered (Fig. 4(c)). This series of measurements, complete with preparations and observations, was reiterated $2000 / n$ times.

The recorded data may be processed in two ways: (i) Only those series of results are considered to represent survival of the ion in its initial state 0 , that include no "on" result with any of the $n$ observations. The number of these series, normalized by the total number of series, approaches the probability of $n$-times survival

$$
P_{00}(n)=\cos ^{2 n}(\pi / 2 n) .
$$

This evaluation is equivalent to discarding all series from the ensemble of survival histories as soon as an "on" result shows up in any of the $n-1$ intermediate observations. The remaining subset contains series with $n-1$ times "off", and with both the results "off" and "on" only of the final probing, such that their sum approaches

$$
P_{00}(n)+P_{01}(n)=\cos ^{2 n-2}(\pi / 2 n) .
$$

The probabilities $P_{i j}(n)$ characterize "selective" measurements, where, $i=0$ indicates the equal outcome " 0 " of the first $n-1$ measurements (out of $n$ ), and $j$ that of the last one. The normalized numbers of series of $n$ measurements that show $n$-fold survival (the frequencies of survival) are shown as dark grey bars in the histogram of Fig. 5. The probability of $n$-fold survival calculated from Eq. (2) is shown as black bars. Also shown, as light grey bars, are the recorded data with the probe pulses missing. The corresponding probability should vanish since effective $\pi$ pulses of the driving microwave have been applied to the ion, which are supposed to warrant unit transition probability, and zero probability of survival, as long as decoherence is negligible.

(ii) All results of intermediate probing might be ignored, and the results of the final probing normalized by the entire number of series. The latter results would represent "non-selective" measurements, and the corresponding probability of survival is

$$
P_{00}^{(n)}=\frac{1}{2}\left(1+\cos ^{n}(\pi / n)\right) .
$$

These values are shown by white bars underlying the black ones. A scheme of non-selective measurements has been used in previous experimental work $[13,14]$. However, this strategy is not suitable for verifying QZE [15].

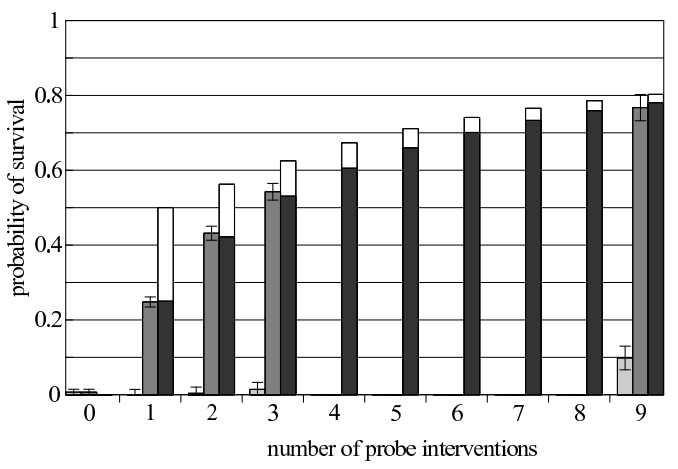

Figure 5: Probability of survival in the "off" state with intermediate probing (dark grey bars), and with no probing (light grey bars), vs. number $n$ of $\pi$-pulse partition. The probability is evaluated by counting as "favourable" those measurements that show only "off" results in each of the $n$ observations. Driving time 2.9 $\mathrm{ms}$, probing time $3 \mathrm{~ms}$. Probability of survival in a "selective" measurement, calculated after Eq. (2) (black bars). If evaluated from the entire ensemble of results, including intermediate "on" results, the probability of survival would represent a non-selective measurement. This probability is calculated after Eq. (4) (white bars).

The observed probabilities of survival are based on detected rates of occurrence of series indentified as "favourable", i.e. seemingly obeying the required conditions, in particular $n$ successive "off" detections per series. These rates have been corrected for initial faulty ion preparation ( $18 \%$ mean value) and false detection of one of the $n$ results of photon counting in a given series of $\pi / n$ pulses. The latter error was determined as follows: The probability distributions of the counting rates of "on" (1) and "off" (0), vs the counted "number of photons", are approximately Poissonian and overlap each other. 
Their distinction is optimum when pulses that contain less than two photon counts are identified as "off" results (0). In fact, the "off" distribution overlaps this threshold by $2 \%$ ( mean value). This overlap is identified as the risk of an individual false detection, from which the rate of misinterpreting a series of $n$ measurements was determined. Multiple false detection within a series was ignored. The error bars of the recorded data represent the variances of the on-off binomial distributions.

The measured and corrected frequencies of survival of the ion's state upon the action of the $n$ times fractionated $\pi$ pulse and intertwined probe interventions vanish at $n=0$ and increase to $77 \%$ at $n=9$. They agree with the values of the probability for selective observations, calculated from Eq. (2) (black bars). Moreover, they are at variance with probability values for non-selective observations, calculated according to Eq. (4). This finding proves the QZP on an individual quantum system without any recurrence to relaxation.

In principle, an observed set of data corresponding to the latter probabilities $P^{(n s)}$ could be derived from the recorded data by simply ignoring the results of $n-1$ probe interventions and relying only to the final $n$th result. Unfortunately, these data include intermediate "on" results that are affected by spurious Zeeman pumping of the ion, by the probe light, into the sub-levels $m_{F}= \pm 1$, outside the considered two-level system $F=0,1, m_{F}=0$. This drawback could be avoided when optically repumping the ion by suitably polarized light during the probe interventions. Note that the results of such a non-selective strategy of evaluation of the measurements are supposed to agree with those of a previous experiment on an ensemble of particles [13,14].

The probability of survival with lacking probe interventions vanishes, except when driving with highly fractionated $\pi$-pulses $(n=9)$ and the concomitant long duration of this pulse series. This $10 \%$ survival probability indicates the inset of some dephasing of the interaction of microwave radiation and spin moment.

In order to better appreciate the role of decoherence, another set of measurements was performed with the pulse length of the intermissions between the fractionated driving pulses extended to $5 \mathrm{~ms}$, but with the probe light missing. The overall duration of a series of measurements now took substantially more time, and dephasing - as indicated by a finite rate of survival upon the $\pi$-pulse excitation considerably increased.

\section{Conclusions}

In summary, we have performed two versions of an experiment on a single ion determined for the demonstration of the quantum Zeno effect with no direct interaction of quantum object with probe radiation and meter, and by null results, i.e., of the "quantum Zeno paradox" [7]. The ground-state hyperfine transition $F=0 \rightarrow 1$ of an individual ${ }^{171} \mathrm{Yb}^{+}$ion was alternatingly driven by resonant microwave pulses of preset pulse area, and probed by laser pulses that did or did not excite resonance scattering recorded by a photon counter. Only those series of driving and probing were counted as "favourable" for the evaluation of the probability of survival that did not show excitation of resonance scattering with the probing.

This experiment is distinguished from a previous one [8] which required the weak spontaneous decay of the "off" state to be modelled, by the complete absence of relaxation with the sequences of results that show no scattering ("off"). These measurements on the ion initially in the $F=0$ state are free of reaction from the meter ("quantum nondemolition", QND [4]). Moreover, they are also free of relaxation. When the ion is in its $F=1$ state, there is no intrinsic relaxation either, but appreciable decoherence is generated when optically pumping the ion, by the probe light, into Zeeman states unaffected by the driving radiation.

The second version of the experiment resembles Cook's suggestion [13]; it includes, however, two important improvements: the use of an individual atomic ion as the quantum system, and the laserexcited scattering being monitored during each of the probing pulses. Thus, the micro-state of the ensemble of observations becomes completely documented, in contrast with measurements on an ensemble of particles. This feature allows one to generate a selective measurement on the individual quantum object, as is required for an unequivocal demonstration of QZP: Only these characteristics allow one to identify back-and-forth transitions of the quantum object during a series of driving $\pi / n$ pulses applied to it, as well as the absence of anti-correlated transitions in two or more individual quantum systems of an ensemble [15]. Consequently, one is able to distinguish the effect of the measurements, i.e., of the entangled quantum system and probe, from that of physical intervention of the probe upon the quantum object [7].

\section{Acknowledgements}

This work was supported by the Hamburgische Wissenschaftliche Stiftung, and by the Deutsche Forschungsgemeinschaft. 


\section{References}

[1] L.A. Khalfin, Pis'ma Zh. Eksp. Teor. Fiz. 8, 106 (1968), [JETP Lett. 8, 65 (1968)].

[2] L. Fonda, G.C. Ghirardi, A. Rimini, R. Weber, Nuovo Cimento A 15, 689 (1973).

[3] B. Misra, E.C.G. Sudarshan, J. Math. Phys. (N.Y.) 18, 756 (1977).

[4] V.B. Braginsky, F.Ya. Khalili, Quantum Measurement, Cambridge University Press, Cambridge, MA, 1992.

[5] T.P. Spiller, Phys. Lett. A 192, 163 (1994).

[6] O. Alter, Y. Yamamoto, Phys. Rev. A 55, 2499 (1997).

[7] D. Home, M.A.B. Whitaker, Annals of Physics 258, 237 (1997).

[8] Chr. Balzer, R. Huesmann, W. Neuhauser, P.E. Toschek, Optics Commun. 180, 115 (2000).

[9] P.E. Toschek, Ch. Wunderlich, Eur. Phys. J. D 14, 387 (2001).

[10] Chr. Balzer, Th. Hannemann, D. Reiss, W. Neuhauser, P.E. Toschek, Chr. Wunderlich, Laser Phys. 12,729 (2002).

[11] R. Huesmann, Ch. Balzer, Ph. Courteille, W. Neuhauser, P.E. Toschek, Phys. Rev. Lett. 82, 1611 (1999).

[12] See, e.g. U. Fano, L. Fano, Physics of Atoms and Molecules, The University of Chicago Press, Chicago, 1970, Also; J. von Neumann, Mathematische Grundlagen der Quantenmechanik, Springer, Berlin, 1932.

[13] R. Cook, Phys. Scripta T 21, 49 (1988).

[14] W.M. Itano, D.J. Heinzen, J.J. Bollinger, D.J. Wineland, Phys. Rev. A 41, 2295 (1990); ibid. 43, 5168 (1991).

[15] H. Nakazato, M. Namiki, S. Pascazio, H. Rauch, Phys. Lett. A 217, 203 (1996). 